\title{
A UNIFORMLY STRONGLY PRIME RADICAL
}

\author{
D. M. OLSON \\ (Received 27 February 1986; revised 28 July 1986) \\ Communicated by R. Lidl
}

\begin{abstract}
The class of all uniformly strongly prime rings is shown to be a special class of rings which generates a radical class which properly contains both the right and left strongly prime radicals and which is independent of the Jacobson and Brown-McCoy radicals.
\end{abstract}

1980 Mathematics subject classification (Amer. Math. Soc.): 16 A 12, 16 A 21.

Keywords and phrases: strongly prime ring, uniformly strongly prime ring, uniformly strongly prime radical, $t$-system.

\section{Introduction}

In 1975 Handelman and Lawrence [5] introduced the concept of right strongly prime rings which are equivalent to the absolutely torsion free rings of Rubin [9]. The right strongly prime radical of a ring $R$ is defined to be the intersection of all right strongly prime ideals of $R$. The dual notion of left strongly primeness determines a left strongly prime radical. An example given by Parmenter, Passman and Stewart [7] showed that these two radicals are distinct. Groenewald and Heyman [4] characterized the right strongly prime radical as the upper radical determined by the special class of all right strongly prime rings. Subsequent work by Parmenter, Stewart and Wiegandt [8] located this radical in the lattice of radical classes.

(c) 1987 Australian Mathematical Society $0263-6115 / 87 \$ A 2.00+0.00$ 
In this paper we define a new radical called the uniformly strongly prime radical which properly contains both the right and left strongly prime radicals. Standard radical theoretic terminology as given in Divinsky [2] will be used and all rings considered will be associative, but need not have identities.

\section{Uniformly strongly prime rings}

Definition 1. A ring $R$ is called Right Strongly Prime if for each nonzero $x \in R$ there exists a finite subset $F_{x}$ of $R$ such that the right annihilator of $x F_{x}$ is zero.

The set $F_{x}$ is called the right insulator of $x$. Handleman and Lawrence worked exclusively with rings with identity; however, Parmenter, Stewart and Wiegandt [8] have shown that the definition is equivalent in a general associative ring to:

Definition 2. A ring $R$ is Right Strongly Prime if each nonzero ideal $I$ of $R$ contains a finite subset $F$ which has right annihilator zero.

Such a finite subset $F$ is called the right insulator of the ideal.

It is clear that every right strongly prime ring is prime. It is also possible to define left strongly prime rings in a manner analogous to that for right strongly prime. Handelman and Lawrence [5] gave an example to show that these two concepts are distinct.

Definition 3. A ring is bounded right strongly prime of bound $n$ (denoted $\left.S P_{r}(n)\right)$ if each nonzero element has an insulator containing no more than $n$ elements and at least one element has no insulator with fewer than $n$ elements.

Definition 4. A ring is called Uniformly Right Strongly Prime if the same insulator may be chosen for each nonzero element.

Since an insulator must be finite, it is clear that every uniformly right strongly prime ring is $S P_{r}(n)$ for some $n$. Again, analogous definitions of bounded left strongly prime and uniformly left strongly prime can be given. As was the case with the strongly prime concept it is possible to find rings which are bounded left strongly prime but not bounded right strongly prime and vice versa [5, Example 1]. However, we find that the concept of uniformly strongly prime is two-sided. 
LEMMA 5. A ring $R$ is uniformly strongly prime if there exists a finite subet $F \subseteq R$ such that for any two nonzero elements $x$ and $y$ of $R$, there exists $f \in F$ such that $x f y \neq 0$.

Proof. Let $R$ be uniformly right strongly prime. Then $R$ has a uniform right insulator $F$ which is a finite set such that for any element $x \in R, x F$ has no nonzero right annihilators. Then, if $x$ and $y$ are any two nonzero elements in $R$, $y$ cannot be in the annihilator of $x F$. Hence there is an $f \in F$ such that $x f y \neq 0$.

For the reverse implication it is easy to see that if the condition is satisfied then for any $x \neq 0$ in $R$, no nonzero element annihilates $x F$ on the right. Hence $R$ is uniformly right strongly prime.

It is obvious that the condition in Lemma 5 is not one-sided; consequently, this condition is equivalent to uniformly left strongly prime as well, and we have:

COROLlaRY 6. $R$ is uniformly right strongly prime if and only if $R$ is uniformly left strongly prime.

DEFINITION 7. We will call a uniformly strongly prime ring US-prime, and an ideal $I$ of a ring $R$ will be called a $U S$-prime ideal of $R$ if $R / I$ is US-prime.

We can establish the following results for US-prime rings which are similar to those which are true for right strongly prime rings.

LEMMA 8. If $R$ is US-prime and $e$ is a nonzero idempotent in $R$ then eRe is US-prime.

Proof. Since $R$ is $U S$-prime, $R$ contains a uniform insulator $F$. Choose $a=e x e$ and $b=e y e$ arbitrary nonzero elements in $e R e$. Then there exists $f \in F$ such that $a f b=$ exefeye $\neq 0$. But then $($ exe $)($ efe $)($ eye $) \neq 0$ and $\{$ efe $\mid f \in F\}$ is a uniform insulator for $e R e$. Hence, $e R s$ is $U S$-prime.

LEMMA 9. For any $n, R$ is US-prime if and only if $M_{n}(R)$ is $U S$-prime.

Proof. Let $R$ be $U S$-prime with insulator $F$. Let $F^{\prime}=M_{n}(F \cup\{0\})$. Then $F^{\prime}$ is a finite set in $M_{n}(R)$. If $A, B \in M_{n}(R)$ are nonzero, then $A$ has a nonzero entry, $a_{i j}$ and $B$ has a nonzero entry, $b_{r s}$. Since $a_{i j}, b_{r s} \in R$ there exists $f \in F$ such that $a_{i j} f b_{r s} \neq 0$. Let $E_{j r}(f)$ be the elementary matrix with $f$ in the $j, r$ position and zeros elsewhere. Then $A E_{j r}(f) B$ has $a_{i j} f b_{r s}$ in the $i, s$ position and hence is nonzero. Sinces $E_{j r}(f) \in F^{\prime}$, we see that $F^{\prime}$ is an insulator for $M_{n}(R)$ and, hence, $M_{n}(R)$ is US-prime. Conversely; suppose, for some $n, M_{n}(R)$ is 
$U S$-prime with insulator $F^{\prime}$. Let $a, b \in R$ be nonzero. If we let $A$ be the matrix with $a$ in the 1,1 position and zeros elsewhere; $B$ the matrix with $b$ in the 1,1 position and zeros elsewhere; then, $A$ and $B$ are nonzero elements of $M_{n}(R)$. Hence, there is an element $D \in F^{\prime}$ such that $A D B$ is nonzero. This requires an entry $d$ in $D$ such that $a d b \neq 0$. So, if we let $F=\{r \mid r$ is an entry in a matrix in $\left.F^{\prime}\right\}$ we see that $F$ becomes an insulator for $R$. Hence $R$ is $U S$-prime.

Lemmas 8 and 9 allow one to conclude that $U S$-primeness is a Morita invariant on the category of associative rings.

THEOREM 10. A right order in a US-prime ring is US-prime.

Proof. Let $R$ be a right order in $S$, a $U S$-prime ring. Let $F=\left\{f_{1}, f_{2}, \ldots, f_{k}\right\}$ be a uniform insulator for $S$. Without loss of generality we may assume $f_{i} \neq 0$ for all $i$. Then, since $R$ is an order in $S$, there exists $t_{1}$, regular in $R$, such that $f_{1} t_{1} \neq 0$ and $f_{1} t_{1} \in R$. But since $t_{1}$ is not a right zero divisor, $f_{2} t_{1} \neq 0$ and there exists a regular element $t_{2} \in R$, such that $\left(f_{2} t_{1}\right) t_{2} \in R$. Continuing, we get $f_{k} t_{1} t_{2} \ldots t_{k} \neq 0$ and $f_{k} t_{1} t_{2} \ldots t_{k} \in R$. Let $t=t_{1} \ldots t_{k}$. Then $t \in R$ since each $t_{i} \in R ; t$ is regular, and $f_{i} t \in R$ for all $i$. Let $F^{*}=\left\{f_{1} t, f_{2} t, \ldots, f_{k} t\right\}$. If $x$ and $y$ are nonzero elements of $R$, then $t y \neq 0$ and since $F$ insulates $S$, there exists $f_{i} \in F$ such that $x f_{i}(t y) \neq 0$ or $x\left(f_{i} t\right) y \neq 0$. Thus, $F^{*}$ is a uniform insulator for $R$ and $R$ is $U S$-prime.

THEOREM 11. Every prime ring with identity can be embedded in a US-prime ring.

Proof. The proof of this result for right strongly prime rings [3, Theorem 3.2] will suffice, if one notices that the ring constructed there has a uniform insulator.

LEMMA 12. If $R$ is US-prime, then any essential extension of $R$ is US-prime.

Proof. Let $S$ be an essential extension of $R$ and let $F$ be the insulator of $R$. It is easy to show that if $x$ is a nonzero element of $S$ then $x F \neq 0$ and $F x \neq 0$, for if not, then $x \in \operatorname{ann}(R)$ which is impossible since $R$ is essential in $S$ and $U S$-prime. So, suppose $s$ and $t$ are nonzero elements of $S$. Then there exist $f_{1}, f_{2} \in F$ such that $s f_{1} \neq 0$ and $f_{2} t \neq 0$. But $s f_{1}$ and $f_{2} t$ are in $R$ so there exists $f \in F$ such that $s f_{1} f f_{2} t \neq 0$. Hence $\left\{f_{1} f f_{2} \mid f_{1}, f, f_{2} \in F\right\}$ is a uniform insulator for $S$ and $S$ is $U S$-prime. 
Now, we shall give a characterization of a $U S$-prime ideal along the lines of the characterization of a prime ideal as an ideal which is a complement to an $m$-system.

Definition 13. A subset $M$ of a ring $R$ is called a $t$-system if there exists a finite set $F \subseteq R$ such that for any two elements $x, y \in M$ there exists $f \in F$ such that $x f y \in M$.

$F$ will be called the insulator of $M$. The empty set will be a $t$-system by definition. The concept of a $t$-system is a somewhat stronger concept than that of an s-system used by Groenewald and Heyman [4] to characterize right strongly prime ideals.

THEOREM 14. An ideal $P$ is a US-prime ideal of a ring $R$ if and only if $R \backslash P$ (the complement of $P$ in $R$ ) is a t-system.

Proof. Let $P$ be an ideal of $R$. Suppose $R \backslash P$ is a $t$-system. Consider $R / P$. If $x+P$ and $y+P$ are nonzero elements of $R / P$ then $x$ and $y$ are in $R \backslash P$. Let $F$ represent the insulator of $R \backslash P$. Then, there exists $f \in F$ such that $x f y \in$ $R \backslash P$. That is $(x+P)(f+P)(y+P)=x f y+P \neq P$. Hence $\{f+P \mid f \in F\}$ is a uniform insulator for $R / P$ and $R / P$ is $U S$-prime. Thus $P$ is a $U S$-prime ideal of $R$.

Conversely, let $P$ be a $U S$-prime ideal of $R$. Then $R / P$ is $U S$-prime. Let $F^{*}=\left\{f_{1}+P, f_{2}+P, \ldots, f_{k}+P\right\}$ be a uniform insulator for $R / P$ for some particular choice of the $f_{i}$. Choose $x, y \in R \backslash P$. Then $x+P$ and $y+P$ are nonzero elements in $R / P$ and hence there exists $f_{1}+P \in F^{*}$ such that $(x+P)\left(f_{1}+P\right)(y+P)=x f_{1} y+P \neq P$. Then $x f_{1} y \in R \backslash P$ and $F=$ $\left\{f_{1}, f_{2}, \ldots, f_{k}\right\}$ is an insulator for $R \backslash P$ and hence $R \backslash P$ is a $t$-system as desired.

We can use this characterization to obtain the following useful lemma.

LEMMA 15. If $A$ and $P$ are ideals of $R$ with $P$ a US-prime ideal, then $P \cap A$ is $a$ $U S$-prime ideal of $A$.

Proof. Consider $a, b \in A \backslash P$. Since $P$ is a $U S$-prime ideal of $R$ we know $R \backslash P$ is a $t$-system with an insulator which we shall call $F$. Thus, for any fixed element $r \in A \backslash P \subseteq R \backslash P$ there exist $f_{1} \in F$ such that $a f_{1} r \in R \backslash P$ and $f_{2} \in F$ such that $\left(a f_{1} r\right) f_{2} b \in R \backslash P$. But, since $a \in A, a f_{1} r f_{2} b \in A \backslash P$, and, since $r \in A, f_{1} r f_{2} \in A$ : Thus the set $\left\{f_{i} r f_{j} \mid f_{1}, f_{j} \in F\right\}$ is a finite insulator for $A \backslash P$ and $P \cap A$ is a $U S$-prime ideal of $A$. 


\section{The $U S$-prime radical}

If $R$ is a ring, the right strongly prime radical of $R$ is the intersection of all its right strongly prime ideals [5]. Groenewald and Heyman [4] characterized this radical as a special radical and Parmenter, Stewart and Weigandt [8] have named it the Groenewald-Heyman radical ( $G H$-radical). In truth, this radical should be called the right $G H$-radical, since there is also a left $G H$-radical and these two radical classes are incomparable [7]. We now define a radical class which contains both the left $G H$-radical and the right $G H$-radical. Recall that a class $\mathscr{M}$ of rings is special in the sense of Andrunakievic [1] if it is a hereditary class of prime rings satisfying

$$
B \in \mathscr{M}, B \subseteq R \text { and ann }(B)=0 \text { implies } R \in \mathscr{M} \text {. }
$$

It was shown in [6] that condition (1) may be replaced by $\left(1^{\prime}\right)$.

$$
\text { Every ring having an essential ideal in } \mathscr{M} \text { is itself in } \mathscr{M} \text {. }
$$

Let $M$ be the class of all $U S$-prime rings.

THEOREM 16. $M$ is a special class of rings.

PROOF. All US-prime rings are strongly prime and hence prime. The hereditary property follows easily from Lemma 15 and the fact that property $\left(1^{\prime}\right)$ is satisfied follows from Lemma 12.

As a consequence of Theorem 16, $M$ generates a special upper radical class $\mathscr{U}(M)$ which we shall call the uniformly strongly prime radical and denote by $U S$. A property of special radical classes is the following.

COROLlaRY 17. For any ring $R, U S(R)=\bigcap\{I \mid I \subseteq R$ and $R / I \in M\}$.

Let the right (left) $G H$-radical be denoted by $s_{\rho}\left(s_{\lambda}\right)$. Then, $s_{\rho}\left(s_{\lambda}\right)$ is the upper radical generated by the class of all right (left) strongly prime rings. Since every $U S$-prime ring is both right strongly prime and left strongly prime, we have the following result.

THEOREM 18. $s_{p} \varsubsetneqq U S$ and $s_{\lambda} \varsubsetneqq U S$.

Proof. The inclusions are clear and since Parmenter, Passman and Stewart [7] have given an example to show that $s_{\rho} \neq\left(s_{\rho} \cap s_{\lambda}\right) \neq s_{\lambda}$, the strictness becomes obvious. 


\section{The lattice of radical classes}

In an effort to locate the radical $U S$ in the lattice of radical classes we first observe that the right (left) strongly prime radical $S_{\rho}\left(S_{\lambda}\right)$ is strictly contained in $U S$ by Theorem 18. Also, since each ring having no zero divisors is $U S$-prime, the radical $U S$ is contained in $N_{g}$, the generalized Nil radical. This containment is strict since the ring $M_{2}(F), F$ a field, is $N_{g}$-radical but $U S$-semisimple. Further positioning is given by

THEOREM 19. US is independent of the Jacobson radical, J, and the BrownMcCoy radical, $G$.

Proof. Goodearl, Handelman, and Lawrence [3, Example 2.3] have given an example of a ring $R$ which is a simple ring with identity which is not bounded strongly prime. Thus, $R$ is not $U S$-prime. Since $R$ is simple, we see that $R \in U S$ but, since $R$ also has identity, $R$ must be $G$-semisimple and hence, $J$-semisimple. On the other hand, the ring

$$
W=\left\{\frac{2 n}{2 m+l} \mid n, m \in Z \text { and }(2 n, 2 m+1)=1\right\}
$$

in Divinsky [2, Example 10, page 103] is a ring which is $J$-radical (and hence $G$-radical). However, $W$ is $U S$-prime since it is a domain, and thus, $W \notin U S$.

The above examples together with results in Parmenter, Stewart and Wiegandt [8] allow us to positon US in the lattice of radicals as in the following diagram.

In the diagram $B$ is the lower Baer radical, which is equal to the prime radical, and $L$ is the Levitski radical.

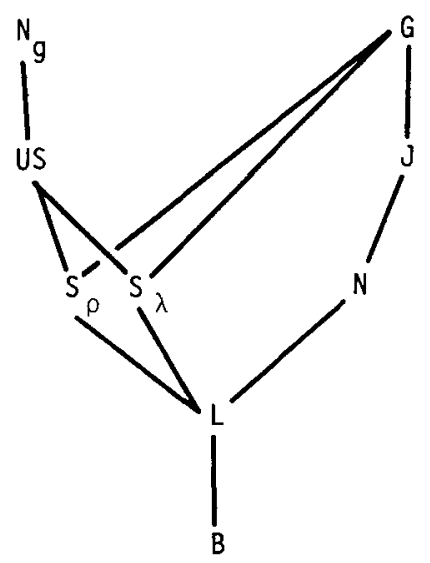




\section{References}

[1] V. A. Andrunakievic, 'Radicals in associative rings I', Amer. Math. Soc. Transl. Ser. 252 (1966), 95-128.

[2] N. Divinsky, Rings and radicals (University of Toronto Press, Toronto, 1965).

[3] K. R. Goodearl, D. Handelman and J. Lawrence, Strongly prime and completely torsion free rings (Carleton Mathematical Series No. 109, Carleton University, Ottowa 1974).

[4] N. J. Groenewald and G. A. P. Heyman, 'Certain classes of ideals in group rings Ir', Comm. Algebra 9 (1981), 137-148.

[5] D. Handelman and J. Lawrence, 'Strongly prime rings', Trans. Amer. Math. Soc. 211 (1975), 209-223.

[6] G. A. P. Heyman and C. Roos, 'Essential extensions in radical theory for rings', J. Austral. Math. Soc. (Ser. A) 23 (1977), 340-347.

[7] M. M. Parmenter, D. S. Passman and P. N. Stewart, 'The strongly prime radical of crossed products', Comm. Algebra 12 (1984), 1099-1113.

[8] M. M. Parmenter, P. N. Stewart and R. Wiegandt, 'On the Groenewald and Heyman strongly prime radical', Quaestiones Math. 7 (1984), 225-240.

[9] R. A. Rubin, 'Absolutely torsion free rings', Pacific J. Math. 46 (1973), 503-514.

John Carroll University

University Heights

Ohio 44118

U.S.A. 